\title{
Epidemiological, Clinical and Pathological Features of Primary Cardiac Hemangiosarcoma in Dogs: A Review of 51 Cases
}

\author{
Shinya YAMAMOTO ${ }^{1}$, Katsuichiro HOSHI'), Atsushi HIRAKAWA ${ }^{3)}$, Syuuichi CHIMURA ${ }^{4)}$, Masayuki KOBAYASHI ${ }^{1)}$ \\ and Noboru MACHIDA ${ }^{1) *}$ \\ 1) Laboratory of Veterinary Clinical Oncology, Tokyo University of Agriculture and Technology, 3-5-8 Saiwai-cho, Fuchu, Tokyo \\ 183-8509, Japan \\ ${ }^{2)}$ Mitsuke Animal Hospital, 5-35-29 Imamachi, Mitsuke, Niigata 954-0111, Japan \\ ${ }^{3)}$ Pet Clinic Hallelujah, 2544-1 Nakabaru, Kasuya, Kasuya-gun, Fukuoka 811-2304, Japan \\ 4) Chimura Veterinary Hospital, 20-13 Minamikarooke, Nakahonmachi, Iwakura, Aichi 482-0042, Japan
}

(Received 6 February 2013/Accepted 16 June 2013/Published online in J-STAGE 28 June 2013)

ABSTRACT. In the study presented here, we aimed to describe the epidemiological, clinical and pathological findings of 51 canine cases with histologically-verified diagnoses of primary cardiac hemangiosarcoma (HSA). The medical data for each dog, including signalment, presenting complaints, physical examination findings, results of various diagnostic testing performed and method of treatment, were checked. In addition, all 51 cases were re-examined pathologically. The tumor occurred most frequently in older Golden Retrievers, followed by Maltese dogs and Miniature Dachshunds. Mass lesions of HSA were found more commonly in the right auricle (RAu) (25/51) and right atrium (RA) $(21 / 51)$, and the RA masses were significantly $(P<0.001)$ larger than the RAu masses. The echocardiographic detection rate of masses in the RAu group $(60 \% ; 15 / 25)$ was significantly lower than that in the RA group $(95 \% ; 20 / 21)$. Survival time was significantly $(P<0.05)$ longer for $5 \mathrm{dogs}$ that received adjuvant chemotherapy after tumor resection than for 12 dogs that did not. In this series, the Maltese (9/51) and Miniature Dachshund (7/51), as well as the Golden Retriever, were represented more frequently than other breeds. The lower echocardiographic detection rate of RAu masses compared with RA masses may be related to tumor size and/or location. The significantly longer survival time for dogs receiving adjuvant chemotherapy indicates that postoperative chemotherapy could be useful for dogs with cardiac HSA.

KEY WORDS: adjuvant chemotherapy, cardiac hemangiosarcoma, epidemiology, survival time.

doi: 10.1292/jvms.13-0064; J. Vet. Med. Sci. 75(11): 1433-1441, 2013

Hemangiosarcoma (HSA) is an aggressive, malignant tumor that arises from either the vascular endothelium or endothelial precursor cells [20]. Primary HSA can develop in any vascularized site in the body, but the most frequent sites of origin include the spleen, right atrium and auricle, subcutaneous tissues and liver $[4,8,14,33]$. This tumor metastasizes easily to distant organs via hematogenous routes, because it has ready access to the systemic circulation, the lungs being the most frequently affected site [23, 29].

Tumors involving the heart occur infrequently in dogs with an overall incidence of $0.19 \%$ according to a database survey [32]. HSA is the most common type of cardiac tumor affecting dogs, representing $69 \%$ of all cardiac tumors identified according to histologic type [32]. Its incidence is approximately 10 -fold higher than the second most common cardiac tumor, aortic body tumor [32]. There appears to be a higher incidence of cardiac HSA in German Shepherd dogs (GSs) and Golden Retrievers (GRs) [1, 2, 4, 15, 19, 24, 27, $30]$ with no apparent sex predilection $[1,30]$. In most cases, primary HSA is located in the right atrium (RA) or auricle

*Correspondence to: Machida, N., Laboratory of Veterinary Clinical Oncology, Tokyo University of Agriculture and Technology, 3-5-8 Saiwai-cho, Fuchu, Tokyo 183-8509, Japan.

e-mail:machida@cc.tuat.ac.jp

(C)2013 The Japanese Society of Veterinary Science
(RAu) $[1,2,13,19,24]$, but occasionally the tumor may arise in the left-sided cardiac chambers $[1,11,19]$.

Presenting signs of cardiac HSA are usually related to pericardial effusion (PE), cardiac tamponade and signs of rightsided heart failure, including lethargy, collapse, abdominal distention, exercise intolerance and dyspnea and cough [2, $9,29,30,33]$. Common abnormalities on physical examination include muffled heart sounds, tachycardia, pale mucous membranes, weak femoral pulses, an abdominal fluid wave and increased respiratory effort [2, 29, 30, 33]. Common laboratory findings include neutrophilia, mild regenerative anemia and large numbers of circulating nucleated and immature red blood cells [2, 17, 22, 29]. Two-dimensional echocardiography is reportedly useful for detection of cardiac masses $[12,22]$; however, reports demonstrating its diagnostic value are limited.

Treatment for cardiac HSA in dogs may include medical and surgical options, although a diagnosis of cardiac HSA generally indicates an extremely poor prognosis [30]. By the time of diagnosis, the tumor has usually metastasized, which may lead owners to opt for palliative treatment, such as repeated pericardiocentesis with or without adjuvant chemotherapy $[6,30]$. Pericardiocentesis is usually associated with marked clinical improvement, but clinical signs of cardiac tamponade typically recur within a few days, often resulting in death or prompting euthanasia [30]. More aggressive approaches to the treatment of cardiac HSA include 
various combinations of pericardectomy, tumor resection and chemotherapy [30, 33]. If identified early without gross evidence of multiple organ metastases, the primary tumor can sometimes be removed surgically, particularly when it is localized to the RAu [10]. Chemotherapy, especially with doxorubicin, following tumor resection may prolong survival, but the benefit appears to be small [33].

In the study presented here, we aimed to clarify the epidemiological, clinical and pathological features of 51 canine cases of primary cardiac HSA and to determine the survival times of dogs with right atrial or auricular HSA treated by pericardectomy and tumor resection with or without adjuvant chemotherapy.

\section{MATERIALS AND METHODS}

Animals: During the period 2002 to 2011, 55 dogs in which primary cardiac HSA had been diagnosed clinically were submitted to the Department of Veterinary Clinical Oncology, Tokyo University of Agriculture and Technology, for postmortem examination. Four of the dogs, which had been euthanatized without treatment at the time of diagnosis, were excluded, leaving 51 dogs as the study materials (Table 1). The medical data obtained for each dog included signalment, presenting complaints, findings of physical examination, results of various diagnostic tests and method of treatment. All 51 dogs died of causes related to PE or metastasis from cardiac HSA. Common presenting complaints included lethargy (35 dogs), weakness (29), exercise intolerance (28), anorexia (22), dyspnea and cough (18), collapse (15) and weight loss (12). Common abnormalities on physical examination included muffled heart sounds (36 dogs), pale mucous membranes (27), tachycardia (24), weak femoral pulses (17), increased respiratory effort (14), jugular distention (11) and abdominal distention (6). CBCs and serum biochemical analyses were performed at initial presentation in all 51 cases. Results of CBCs were normal for 19 dogs; the remaining 32 dogs had mild anemia (21), mild leukocytosis (15) or both (4). Serum biochemistry parameters were generally within the normal ranges, although mild increases in the blood urea nitrogen concentration (18) and serum glutamic pyruvic transaminase activity (11) were noted.

Echocardiography: Echocardiography was performed in all 51 dogs using an Aloka Prosound SSD-4000, SSD-5500 or $\alpha 7$ ultrasound machine with a 3.8- to $7.5-\mathrm{MHz}$ sector array transducer (Hitachi-Aloka Medical, Ltd., Tokyo, Japan). Multiple echocardiographic views were obtained to thoroughly evaluate dogs for cardiac masses, including right and left parasternal short-axis and long-axis views. All echocardiographic examinations were performed by a single operator with the dogs in lateral recumbency. Echocardiograms were considered to be positive, if a mass was identified at initial presentation.

Treatment and survival time: For all 51 dogs, clinical staging was performed using the modified TNM World Health Organization (WHO) staging protocol of Aronsohn [1] and Russell et al. [25]. This system is based on tumor size, degree of invasion, lymph node involvement and signs of distant metastasis and is applicable to canine HSA [14]. On the basis of the presence or absence of metastatic disease demonstrated by thoracic radiography and abdominal ultrasonography at initial presentation, $31 \mathrm{dogs}$ that did not have evidence of distant metastasis were classified as having stage-2 HAS, and 20 were classified as having stage-3 HSA (Table 1). There were no dogs with stage-1 HSA at the time of diagnosis.

The 51 dogs were allotted to 1 of 4 groups based on methods of treatment: no treatment - group A (8: stage 2, 2; stage 3,6$)$; medical treatment - group B (26: stage 2, 12; stage 3,14$)$; pericardectomy and tumor resection - group C (12: stage 2,12$)$; and pericardectomy, tumor resection and chemotherapy - group D (5: stage 2, 5) (Table 1). Medical management was attempted in 26 dogs of group B. Treatment regimens included administration of various antibiotics, glucocorticoids, diuretics, digitalis and antiarrhythmic drugs. Nine of the 26 dogs were also treated with intermittent pericardiocentesis, 3 times in 2 dogs, twice in 4 and once in 3. All 12 dogs in group $\mathrm{C}$ underwent subtotal pericardectomy and surgical resection of the RAu or RA mass (Fig. 1). Five dogs in group D all underwent subtotal pericardectomy, surgical resection of the RAu or RA mass and adjuvant chemotherapy. For groups C and D, a right lateral thoracotomy was performed in $10 \mathrm{dogs}$, and a median sternotomy was performed in 7 .

The chemotherapy strategy chosen was 3-week cycles consisting of doxorubicin $\left(30 \mathrm{mg} / \mathrm{m}^{2}\right.$, IV) and cyclophosphamide $\left(100 \mathrm{mg} / \mathrm{m}^{2}, \mathrm{IV}\right)$ on day 1 and vincristine $(0.5 \mathrm{mg} /$ $\left.\mathrm{m}^{2}, \mathrm{IV}\right)$ on days 8 and 15 [7]. The protocol was initiated at the time of suture removal and repeated every 21 days for a maximum of 5 cycles or until disease progression. A physical examination and a $\mathrm{CBC}$ and serum biochemical analysis were performed weekly to detect adverse reactions.

Survival time was defined as the time from the date of clinical diagnosis to death.

Necropsy, histopathology and immunohistochemistry: Complete necropsy was performed on all 51 dogs (necropsy alone 34; tumor resection and necropsy 17). At necropsy, the removed heart was subjected to gross examination, and the exact location of any tumors was identified. It was then placed in $10 \%$ phosphate-buffered formalin for at least 5 days. After fixation, in the $34 \mathrm{dogs}$ that had not undergone tumor resection, mass lesions were excised en bloc and weighed, and the tumor weight-to-body weight ratio (TW/ BW) was calculated (i.e. tumor weight in g/body weight in $\mathrm{kg}$ ). For the remaining 17 dogs that had undergone tumor resection, the resected masses had been weighed at that time. In each case, at least 5 tissue blocks were taken from the cardiac mass and processed by routine methods. Sections $(5 \mu \mathrm{m})$ were stained with hematoxylin and eosin (HE) for light microscopy. In addition, tissue samples were collected from all internal organs, focusing on those with tumorous lesions indicative of metastasis. For the immunohistochemical study, sections were stained by the avidin-biotin-peroxidase method (Vectastain, Vector Laboratories, Burlingame, CA, U.S.A.) using antibodies against the cellular proteins vimentin (1:200, monoclonal, clone MNF 16; Dako, Glostrup, 
Table 1. Clinical and pathological summary of 51 dogs with cardiac hemangiosarcoma

\begin{tabular}{|c|c|c|c|c|c|c|c|c|c|}
\hline \multirow[b]{2}{*}{ Dog } & \multicolumn{6}{|c|}{ UCG } & \multirow[b]{2}{*}{$\begin{array}{l}\text { Survival } \\
\text { (d) }\end{array}$} & \multicolumn{2}{|r|}{ Necropsy (surgery) } \\
\hline & Breed & $\begin{array}{l}\text { Age } \\
\text { (y) }\end{array}$ & Sex & Mass detection & $\begin{array}{l}\text { Clinical } \\
\text { Stage }\end{array}$ & Therapy & & $\begin{array}{l}\text { Location } \\
\text { of mass }\end{array}$ & Metastasis \\
\hline 1 & Golden Retriever & 7 & M & Negative & 3 & A & 1 & $\mathrm{RAu}$ & $\mathrm{Lu}, \mathrm{Li}, \mathrm{Sp}$ \\
\hline 2 & Mixed breed & 8 & M & Positive & 3 & A & 5 & $\mathrm{RV}$ & $\mathrm{Lu}, \mathrm{He}$ \\
\hline 3 & German Shepherd & 9 & $\mathrm{~F}$ & Negative & 2 & $\mathrm{~A}$ & 1 & $\mathrm{RAu}$ & - \\
\hline 4 & Labrador Retriever & 10 & M & Positive & 3 & $\mathrm{~A}$ & 2 & $\mathrm{RAu}$ & $\mathrm{Lu}, \mathrm{Li}, \mathrm{Sp}, \mathrm{He}, \mathrm{Sc}$ \\
\hline 5 & Miniature Dachshund & 12 & M & Positive & 3 & $\mathrm{~A}$ & 1 & $\mathrm{RAu}$ & $\mathrm{Pu}$ \\
\hline 6 & Maltese & 13 & $\mathrm{~F}$ & Positive & 3 & $\mathrm{~A}$ & 14 & $\mathrm{RAu}$ & $\mathrm{Lu}, \mathrm{Li}, \mathrm{Sp}$ \\
\hline 7 & Golden Retriever & 13 & $\mathrm{~F}$ & Positive & 2 & A & 26 & $\mathrm{RAu}$ & $\mathrm{Lu}, \mathrm{Li}, \mathrm{He}, \mathrm{Ki}, \mathrm{Pa}, \mathrm{Sc}, \mathrm{In}, \mathrm{Om}, \mathrm{Di}$ \\
\hline 8 & Golden Retriever & 15 & $\mathrm{~F}$ & Positive & 3 & A & 7 & $\mathrm{RAu}$ & - \\
\hline 9 & Mixed breed & 6 & M & Negative & 2 & $\mathrm{~B}$ & 32 & $\mathrm{RAu}$ & $\mathrm{Lu}, \mathrm{Li}, \mathrm{Sp}, \mathrm{Br}$ \\
\hline 10 & Golden Retriever & 7 & M & Negative & 3 & $\mathrm{~B}$ & 23 & RAu & $\mathrm{Lu}, \mathrm{Li}, \mathrm{Sp}, \mathrm{Pe}$ \\
\hline 11 & Maltese & 8 & $\mathrm{~F}$ & Positive & 3 & $\mathrm{~B}$ & 4 & RA & $\mathrm{Lu}$ \\
\hline 12 & Golden Retriever & 8 & M & Positive & 2 & $\mathrm{~B}$ & 41 & RA & - \\
\hline 13 & Mixed breed & 9 & $\mathrm{~F}$ & Positive & 2 & $\mathrm{~B}$ & 32 & RAu & - \\
\hline 14 & Miniature Dachshund & 9 & M & Positive & 3 & $\mathrm{~B}$ & 1 & RA & $\mathrm{Lu}, \mathrm{Li}, \mathrm{Sp}, \mathrm{Br}, \mathrm{Ki}, \mathrm{In}, \mathrm{Pa}, \mathrm{Om}, \mathrm{Ad}$ \\
\hline 15 & Jack Russell Terrier & 10 & M & Positive & 2 & $\mathrm{~B}$ & 45 & RA & $\mathrm{Lu}, \mathrm{Sp}, \mathrm{He}, \mathrm{Om}, \mathrm{In}, \mathrm{Me}, \mathrm{Ly}$ \\
\hline 16 & Maltese & 10 & M & Positive & 2 & $\mathrm{~B}$ & 124 & RV & $\mathrm{Lu}, \mathrm{He}$ \\
\hline 17 & Welsh Corgi & 11 & $\mathrm{~F}$ & Positive & 3 & $\mathrm{~B}$ & 30 & RA & $\mathrm{Lu}, \mathrm{Li}, \mathrm{Sp}, \mathrm{He}$ \\
\hline 18 & Golden Retriever & 11 & $\mathrm{~F}$ & Positive & 3 & B & 7 & $\mathrm{RAu}$ & $\mathrm{Lu}, \mathrm{Li}, \mathrm{Sp}, \mathrm{Pe}$ \\
\hline 19 & Maltese & 11 & M & Positive & 2 & B & 9 & RA & - \\
\hline 20 & Welsh Corgi & 11 & $\mathrm{~F}$ & Positive & 3 & $\mathrm{~B}$ & 21 & RA & $\mathrm{Lu}, \mathrm{Li}, \mathrm{Sp}$ \\
\hline 21 & Welsh Corgi & 12 & M & Positive & 2 & $\mathrm{~B}$ & 69 & RA & - \\
\hline 22 & Border Collie & 12 & M & Negative & 2 & B & 188 & HB & $\mathrm{Lu}$ \\
\hline 23 & Maltese & 12 & M & Positive & 2 & $\mathrm{~B}$ & 27 & RA & - \\
\hline 24 & Pomeranian & 12 & M & Positive & 3 & B & 17 & RA & $\mathrm{Lu}$ \\
\hline 25 & Maltese & 12 & M & Positive & 3 & B & 5 & RA & $\mathrm{Lu}, \mathrm{Ad}$ \\
\hline 26 & Yorkshire Terrier & 12 & M & Positive & 3 & B & 18 & RA & $\mathrm{Sp}, \mathrm{He}$ \\
\hline 27 & Mixed breed & 12 & M & Positive & 3 & B & 52 & RA & $\mathrm{Lu}, \mathrm{Li}, \mathrm{Sp}$ \\
\hline 28 & Mixed breed & 13 & $\mathrm{~F}$ & Positive & 2 & B & 143 & RA & $\mathrm{Lu}, \mathrm{He}$ \\
\hline 29 & Shetland Sheepdog & 13 & M & Positive & 2 & B & 65 & RA & $\mathrm{Lu}, \mathrm{Li}, \mathrm{Sp}, \mathrm{Ki}, \mathrm{Br}$ \\
\hline 30 & Beagle & 13 & M & Positive & 3 & B & 8 & RA & $\mathrm{Lu}, \mathrm{Li}, \mathrm{Sp}, \mathrm{Br}, \mathrm{Sc}$ \\
\hline 31 & Mixed breed & 14 & $\mathrm{~F}$ & Negative & 3 & B & 22 & $\mathrm{RAu}$ & $\mathrm{Lu}$ \\
\hline 32 & Miniature Dachshund & 16 & M & Negative & 3 & B & 27 & RA & $\mathrm{Lu}, \mathrm{Li}, \mathrm{Sp}, \mathrm{He}, \mathrm{Me}, \mathrm{Sc}$ \\
\hline 33 & Siberian Husky & 16 & M & Negative & 3 & B & 8 & $\mathrm{RAu}$ & $\mathrm{Lu}, \mathrm{Sp}$ \\
\hline 34 & Mixed breed & 16 & $\mathrm{~F}$ & Negative & 2 & B & 51 & $\mathrm{LAu}$ & $\mathrm{Lu}, \mathrm{Ki}, \mathrm{He}, \mathrm{Om}$ \\
\hline 35 & Shih Tzu & 6 & M & Positive & 2 & $\mathrm{C}$ & 100 & RA & $\mathrm{Lu}, \mathrm{He}$ \\
\hline 36 & Labrador Retriever & 7 & M & Positive & 2 & $\mathrm{C}$ & 105 & RAu & $\mathrm{Lu}, \mathrm{Li}, \mathrm{Sp}, \mathrm{Ki}, \mathrm{Me}, \mathrm{Ad}$ \\
\hline 37 & Maltese & 8 & M & Negative & 2 & $\mathrm{C}$ & 47 & $\mathrm{RAu}$ & $\mathrm{Lu}, \mathrm{Sc}$ \\
\hline 38 & Labrador Retriever & 9 & M & Positive & 2 & $\mathrm{C}$ & 113 & RAu & $\mathrm{Lu}$ \\
\hline 39 & Siberian Husky & 9 & M & Positive & 2 & $\mathrm{C}$ & 59 & $\mathrm{HB}$ & $\mathrm{Li}, \mathrm{He}, \mathrm{Om}, \mathrm{Me}, \mathrm{Sc}, \mathrm{Pa}$, In \\
\hline 40 & Golden Retriever & 9 & $\mathrm{~F}$ & Negative & 2 & $\mathrm{C}$ & 82 & $\mathrm{RAu}$ & - \\
\hline 41 & Golden Retriever & 9 & $\mathrm{~F}$ & Positive & 2 & $\mathrm{C}$ & 67 & RAu & $\mathrm{Sp}$ \\
\hline 42 & Golden Retriever & 10 & M & Positive & 2 & $\mathrm{C}$ & 10 & RAu & $\mathrm{He}$ \\
\hline 43 & Maltese & 12 & $\mathrm{~F}$ & Positive & 2 & $\mathrm{C}$ & 90 & $\mathrm{RAu}$ & - \\
\hline 44 & Golden Retriever & 12 & $\mathrm{~F}$ & Positive & 2 & $\mathrm{C}$ & 202 & RAu & $\mathrm{Lu}$ \\
\hline 45 & Miniature Dachshund & 13 & $\mathrm{~F}$ & Positive & 2 & $\mathrm{C}$ & 182 & RA & $\mathrm{Sc}$ \\
\hline 46 & Mixed breed & 13 & M & Negative & 2 & $\mathrm{C}$ & 15 & RAu & - \\
\hline 47 & Miniature Dachshund & 5 & $\mathrm{~F}$ & Negative & 2 & $\mathrm{D}$ & 189 & RAu & $\mathrm{Lu}$ \\
\hline 48 & Miniature Dachshund & 8 & M & Positive & 2 & $\mathrm{D}$ & 127 & $\mathrm{RAu}$ & - \\
\hline 49 & Golden Retriever & 13 & $\mathrm{~F}$ & Positive & 2 & $\mathrm{D}$ & 241 & $\mathrm{RAu}$ & $\mathrm{Lu}$ \\
\hline 50 & Miniature Dachshund & 13 & M & Positive & 2 & $\mathrm{D}$ & 118 & RA & - \\
\hline 51 & Maltese & 14 & M & Positive & 2 & $\mathrm{D}$ & 240 & RA & - \\
\hline
\end{tabular}

M: Male, F: Female, A: No treatment, B: Medical treatment, C: Pericardiectomy and/or tumor resection, D: Pericardiectomy and/or tumor resection and chemotherapy, RAu: Right auricle, RA: Right atrium, HB: Heart base, RV: Right ventricle, LAu: Left auricle, Lu: Lung, Sp: Spleen, Li: Liver, He: Other parts of the heart, Sc: Subcutaneous tissue, Ki: Kidney, Me: Mesentery, In: Intestine, Om: Omentum, Pa: Pancreas, Ad: Adrenal grand, Br: Brain, Pe: Peritneum, Di: Diaphragm, Ly: Lymph node. 

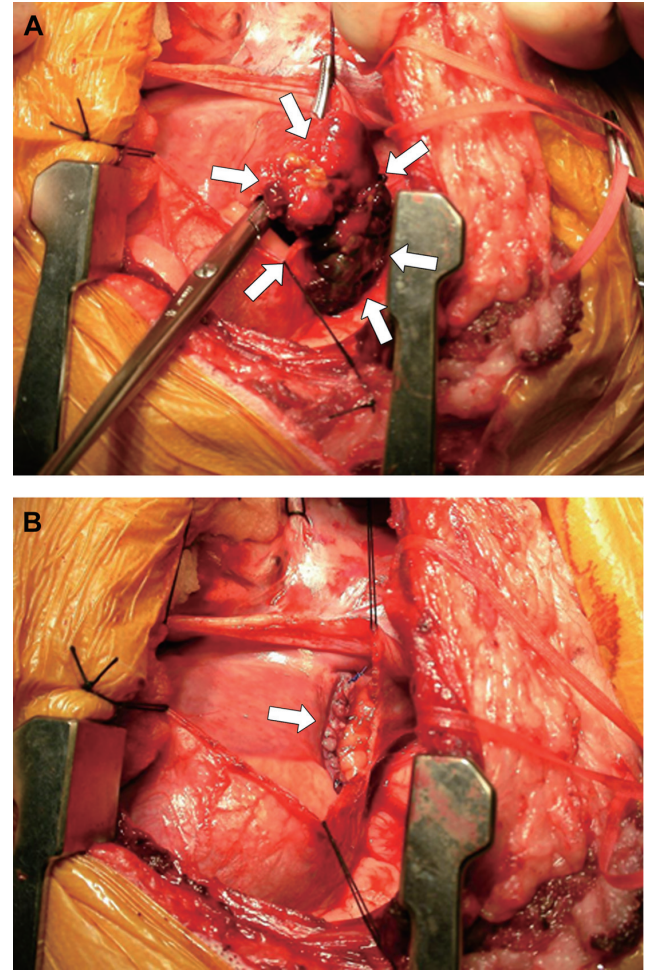

Fig. 1. Intraoperative photographs of cardiac hemangiosarcoma, showing a pedunculated mass located in the right auricle (arrows) (A) and the right atrial wall oversewn with 2 continuous rows of nonabsorbable suture material (arrow) (B).

Denmark), cytokeratin (1:100, monoclonal, clone Vim 3B4; Dako), CD31 (1:40, monoclonal, clone JC70A; Dako) and von Willebrand factor (factor VIII-related antigen) (1:50, monoclonal, clone F8/86; Dako).

Statistical analysis: The age, the body weight, the tumor weight and the TW/BW value were expressed as mean $\pm \mathrm{SD}$. Fisher's exact test was used to evaluate the difference in the echocardiographic detection rate of the masses between dogs with masses involving the RAu (RAu group) and dogs with masses involving the RA (RA group). Kaplan-Meier curves were generated, and the log-rank test was used to compare median survival times between groups A, B and C, and D. Mann-Whitney $\mathrm{U}$ test was used to evaluate the difference in the data for CBCs (WBC, RBC and platelet) or serum biochemistry (ALT, AST, ALP, T-Bil, TP, albumin, BUN and creatinine) between group $\mathrm{C}$ and group D. Student's $t$ test was used to evaluate the difference in the TW/BW value between the RAu and RA groups. For all statistical analyses, standard software was used, and differences of $P<0.05$ were considered to be significant.

\section{RESULTS}

Signalment: The breeds of the dogs enrolled in this study included: GR (11 dogs); Maltese (9); mixed-breed (8); Min-
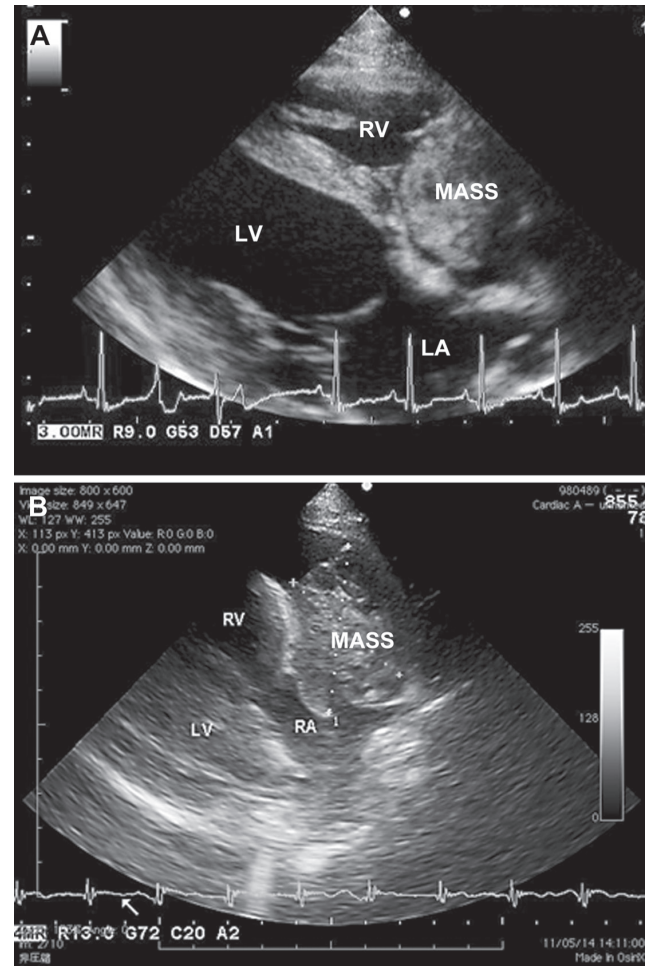

Fig. 2. Two-dimensional echocardiograms (long-axis view from the right parasternal location) of dogs with primary cardiac hemangiosarcoma, showing a cavitary and cystic mass (MASS) associated with the right auricle (A) and a cavitated soft tissue mass (MASS) occupying the right atrial cavity. LA, left atrium; LV, left ventricle; RA, right atrium, RV, right ventricle.

iature Dachshund (7); Labrador Retriever (3); Welsh Corgi Pembroke (3); Siberian Husky (2); and one each of the following: Pomeranian; Yorkshire Terrier; Shih Tzu; Jack Russell Terrier; Shetland Sheepdog; Beagle; Border Collie; and GS (Table 1). The mean age was $10.7 \pm 2.8$ years (range, 5 to 16 years). Males accounted for $63 \%(32 / 51)$ of the cases and females for $37 \%$ (19/51) (Table 1). The mean body weight was $16.5 \pm 11.6 \mathrm{~kg}$ (range, 2.5 to $40 \mathrm{~kg}$ ).

Echocardiography: PE was seen in $42(82 \%)$ of the 51 dogs, and a lesion suggestive of a cardiac mass was detected in $38(75 \%)$. On the basis of sonography, masses were predicted to originate from the RA in 20 dogs (Fig. 2A), RAu in 15 dogs (Fig. 2B), RV in 2 dogs and heart base in 1 dog (Table 1). The sensitivity of two-dimensional echocardiography for detecting tumor-positive dogs was $95 \%(20 / 21)$ for the RA group and $60 \%(15 / 25)$ for the RAu group. Thus, the detection rate for masses involving the RAu was significantly lower than that for tumors involving the RA $(P<0.05)$.

Treatment and survival time: We did not detect any obvious differences or predilections between the groups with respect to breed, age or sex (Table 1). Surgical intervention with pericardectomy and tumor resection was not associated with serious complications that required prolonged hospital- 
A

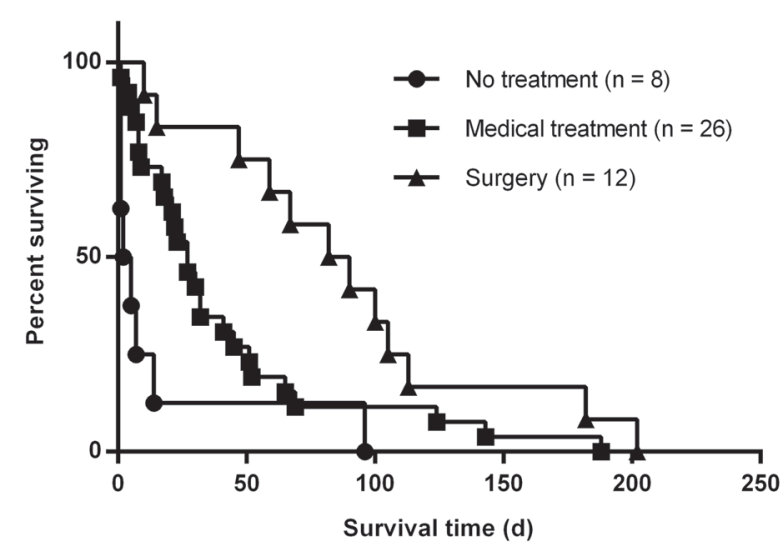

B

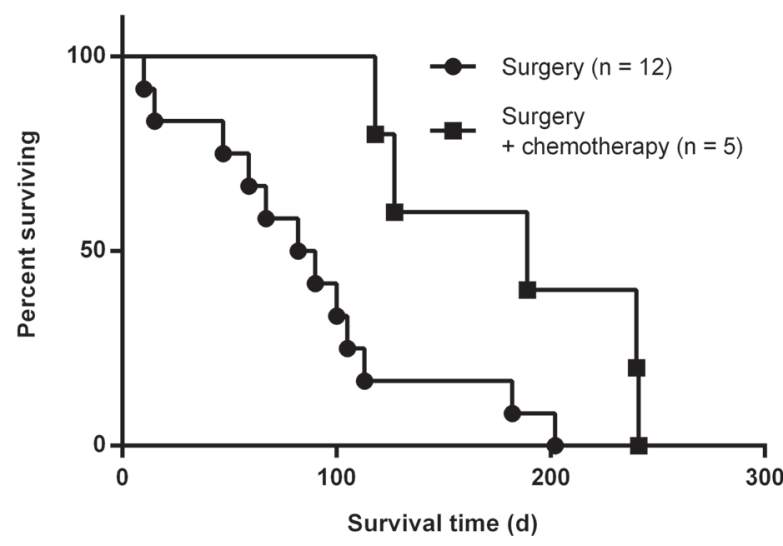

Fig. 3. Kaplan-Meier survival curves for dogs with right atrial or auricular hemangiosarcoma. A, A combination of pericardectomy and surgical resection of the tumor (triangles) was associated with significantly longer survival time, compared with no treatment (circles; $P<0.001$ ) or medical treatment alone (squares; $P<0.05$ ). $\mathrm{B}$, survival was significantly $(P<0.05)$ longer for dogs that received adjuvant chemotherapy after surgery (squares) than for dogs that did not (circles).

ization in any of the dogs. Median survival times were 7.1 days (range, 1 to 26 days) for group A, 27 days (range, 1 to 188 days) for group B, 86 days (range, 10 to 202 days) for group $\mathrm{C}$ and 189 days (range, 118 to 241 days) for group $\mathrm{D}$, respectively (Table 1 and Fig. 3). Statistical analysis indicated that a combination of pericardectomy and surgical resection of the tumor (group C) was associated with significantly longer survival time, compared with dogs that received no treatment (group A; $P<0.001$ ) or medical management alone (group B; $P<0.05$ ) (Fig. 3A). Furthermore, survival time was significantly $(P<0.05)$ longer for dogs that received adjuvant chemotherapy after surgery (group D) than for dogs that did not (group C) (Fig. 3B). No significant differences were identified between group $C$ and group D with regard to pretreatment data for CBCs or serum biochemistry. Four of the five dogs that received postoperative adjuvant chemotherapy (group D) revealed mild leukopenia after the treatment with no significant differences. Other posttreatment data for $\mathrm{CBCs}$ and serum biochemistry in group $\mathrm{C}$ and group $\mathrm{D}$ showed no significant differences. Two of the five dogs developed vomiting and diarrhea presumably associated with doxorubicin administration. Another minimal complication observed in one dog was lethargy for 1 to 2 days after administration of each dose of doxorubicin.

Necropsy, histopathology and immunohistochemistry: Primary mass lesions of the heart were identified in all $51 \mathrm{dogs}$ at surgical biopsy (17) or necropsy (34). The cardiac tumors were confined to the RAu (Fig. 4A) in 25 dogs and to the RA (Fig. 4B) in 21 dogs (Table 1). In 13 of the 21 dogs with RA masses, the masses were solitary and originated from the anterolateral regions of the RA; the smallest and most discrete tumors were located predominantly beneath the epicardium just above the atrioventricular junction (Fig. 5). In the remaining $8 \mathrm{dogs}$, the masses were large and invasive, involving most of the RA. In 5 other dogs, a single mass lesion was located in the right ventricle (RV) in 2 (Fig. 4C), in the heart base in 2 and in the left atrium in one (Fig. 4D) (Table 1). In the 2 dogs with masses widely involving the heart base, the exact anatomical origin of the tumors could not be determined.

The tumors were nodular or botryoidal and poorly circumscribed, and in many cases, there was diffuse infiltration of the wall involving its entire thickness. The tumors ranged from $1 \mathrm{~cm}$ to $15 \mathrm{~cm}$ or more in greatest dimension, but most were approximately 2 to $5 \mathrm{~cm}$. The masses were mottled white/red or dark red depending on the degree of cellularity and the amount of blood in the vascular spaces. Many of them were of loose consistency, because of a considerable amount of blood inside the tumors. The RA masses were often seen to bulge out beyond the epicardium or endocardium, sometimes encroaching upon the RA cavity.

There was a considerable range in the weight of the tumors; the lightest was $0.8 \mathrm{~g}$ and the heaviest $87.2 \mathrm{~g}$ (mean $18.7 \pm 17.6 \mathrm{~g}$ ). The TW/BW value ranged from 0.1 to 1.3 for the RAu group and from 0.7 to 4.8 for the RA group. The mean TW/BW value for the RA group $(2.6 \pm 1.2)$ was significantly higher than that for the RAu group $(0.6 \pm 0.4)$ $(P<0.001)$.

Postmortem study of the 51 dogs showed that 38 (75\%) had metastatic disease. Affected sites included the lungs (33, $87 \%)$, spleen $(17,45 \%)$, liver $(16,42 \%)$, other parts of the heart including the pericardium $(8,21 \%)$, subcutaneous tissues $(6,16 \%)$, kidneys $(5,13 \%)$, mesentery $(4,11 \%)$, intestines $(4,11 \%)$, omentum $(4,11 \%)$, brain $(3,8 \%)$, adrenal glands $(3,8 \%)$, peritoneum $(2,5 \%)$, visceral lymph nodes $(1$, $3 \%)$ and diaphragm $(1,3 \%)$ (Table 1$)$.

The histological picture of the tumors varied within and between dogs, showing varying degrees of vascular differentiation. It ranged from a typical cavernous structure with well-differentiated, spindle-shaped cells on a delicate connective tissue framework forming blood-filled cavities and channels, to a rather solid configuration with poorly differentiated, pleomorphic cells in which cell-lined vascular 

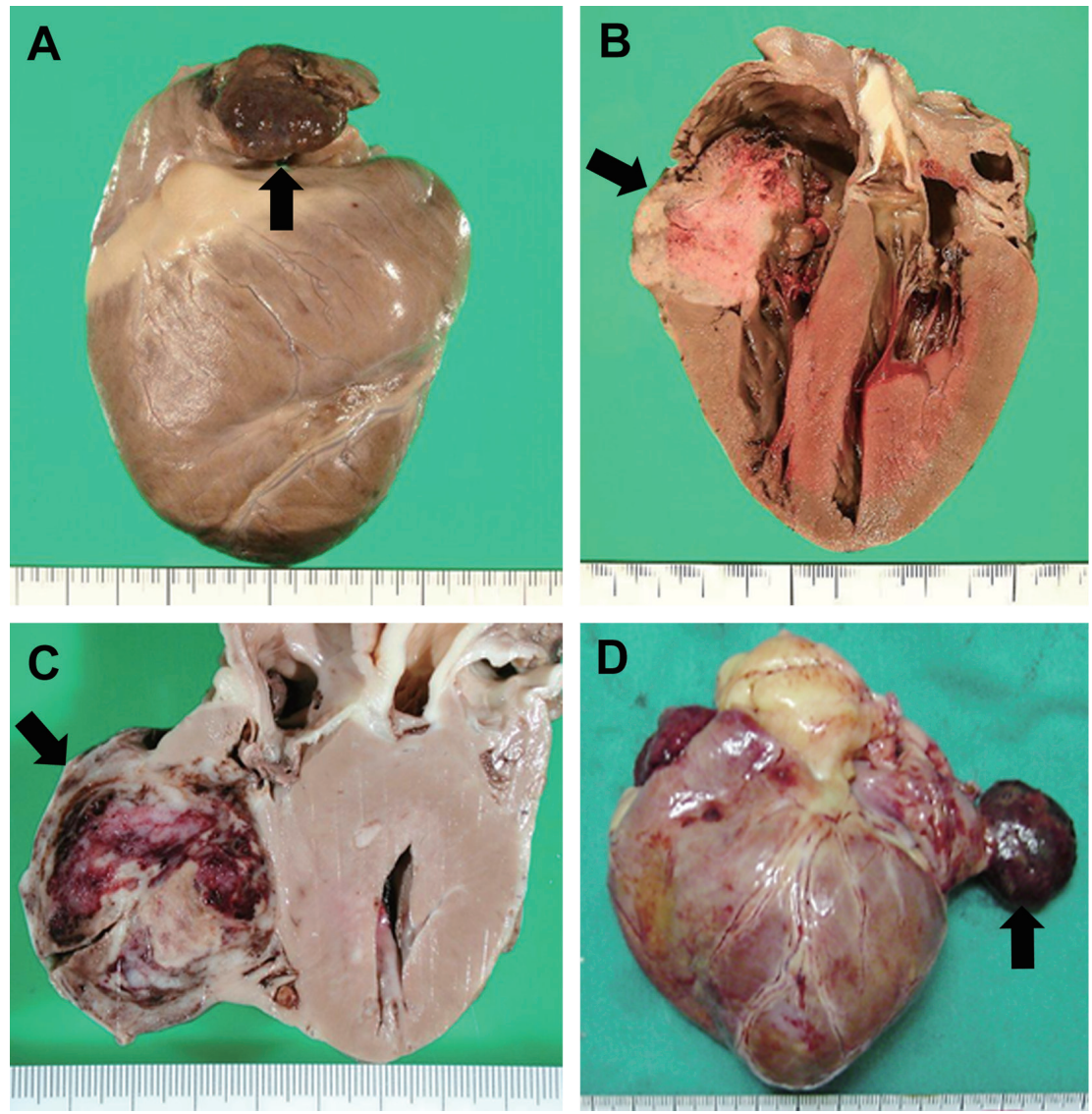

Fig. 4. Right lateral view (A), longitudinal sections (B and C) and ventral view (D) of the heart, showing mass lesions of hemangiosarcoma arising from the right auricle (A), right atrium (B), right ventricle (C) and left atrium. Scale, $1 \mathrm{~mm}$.

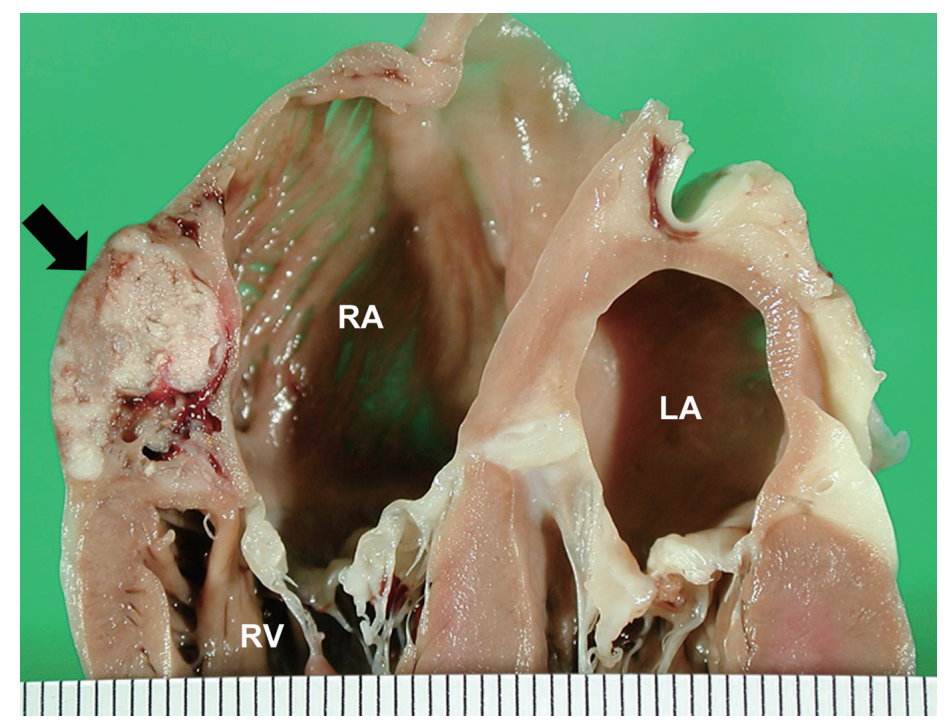

Fig. 5. Heart transected along the long axis, showing hemangiosarcoma arising in subepicardial tissue in the anterolateral region of the right atrium just above the atrioventricular junction. Scale, $1 \mathrm{~mm}$. 
spaces were not obvious. Between these two extremes, there were intermediate forms that displayed an extensive and irregular network of usually fairly narrow spaces and channels. It was not unusual for the same tumor to show diverse forms of differentiation. The extent and intensity of immunohistochemical staining did not vary greatly depending on the histological pattern of the tumor, although there was some variation between different areas in the same section or among different sections in the same case. In all 51 dogs, the tumor cells exhibited moderate-to-strong, diffuse immunoreactivity for vimentin and CD 31 with mild, infrequent positive staining for factor VIII-related antigen; however, they were negative for cytokeratin.

\section{DISCUSSION}

Cardiac HSA is the most commonly reported primary neoplasm of the heart in dogs, occurring most frequently in older GSs and GRs [1, 4, 15, 19, 24, 27, 30] and usually involving the RA or RAu $[1,2,13,19,24]$. The tumor is highly malignant, and widespread hematological dissemination occurs early in the course of the disease [1, 19, 22]. Therefore, it has been considered that treatment for cardiac HSA is challenging and that the tumor has a grave prognosis [30]. In the present study, metastatic disease was found in 38 of $51(75 \%)$ dogs with primary cardiac HSA at necropsy. This figure emphasizes the fact that widespread metastasis of this tumor to other organs is common.

The epidemiologic data in this series of cases were consistent with those of other studies $[1,19,24]$. The mean age of affected dogs in our series was 10.7 years, and none of the dogs was younger than 5 years of age. This age disposition for dogs with cardiac HSA has been recognized throughout the earlier literature $[1,4,19,23,24]$. The total numbers of males and females were 32 and 19, respectively. The male/ female ratio was 1.68 , indicating a slight predominance in males, although we were unable to compare this figure with the proportions of male dogs in hospital populations at large. This information corresponds to other studies that have reported a male sex predilection for canine HSA. [4, 19, 23]. Other studies have indicated no particular sex predilection $[1,30]$, although it has been suggested that spayed females may be at increased risk [32].

Our study sample was too small to allow conclusions to be drawn about breed distribution, but there were 11 GRs in the present series, and this was a breed that had featured in earlier investigations $[1-4,26]$. It was also evident that Maltese (9/51) and Miniature Dachshund (7/51) breeds were represented more frequently than others. Predisposition of these 2 breeds to canine HSA has not been mentioned by any previous reports. It is considered that the high frequency of the tumor in Maltese and Miniature Dachshunds is not attributable to 'Japan strains' of these breeds, but possibly reflects a predisposition of these breeds to certain environmental factors. Although cardiac HSA is reportedly most common in GSs as mentioned above $[1,2,4,19,24]$, such a predilection was not noted in the present series. Such variations may reflect differences in the time frame, geography and breed prevalence of the present and previous studies.

As to the location of primary cardiac HSA, we found that these tumors arose most often in the RAu and RA, but occasionally in the RV, heart base and left atrium. Primary cardiac HSAs are usually located in the RA or RAu [1, 2, $13,19,24]$, and other sites of the heart are affected only occasionally $[1,11,19]$. In the present series, the sites in the heart affected by cardiac HSAs were primarily the same as those reported previously $[1,19,22]$, regardless of breed differences between this and other studies.

The most frequent location of cardiac HSAs in humans is the RA [5], as is the case in dogs [1, 2, 13, 19, 24]. Other types of sarcomas in humans also occur more commonly in the RA than in other parts of the heart [5]. This site is a frequent location for malignant lymphoma in cattle, and it has been reported that foci of undifferentiated connective tissue, often found in the subepicardium of the RA of cattle, correspond to sites of involvement of malignant lymphoma [16]. This embryonic connective tissue is also found in the RA of other animals [16], and it seems possible that it could give rise to various types of sarcomas including HSAs [19]. Interestingly, the majority of RA masses in the present series originated from the anterolateral regions of the RA as solitary masses; the smallest and most discrete tumors were located predominantly beneath the epicardium just above the atrioventricular junction.

Accurate diagnosis of a cardiac mass and defining its location can yield information that is important for therapeutic planning. In the present series, a lesion suggestive of a cardiac mass was detected echocardiographically in 38 (75\%) of the 51 dogs with primary cardiac HSAs. The sensitivity of two-dimensional echocardiography for detection of a mass lesion was significantly lower for RAu than for RA masses. The mean TW/BW value in the RA group was significantly higher than that in the RAu group, indicating that echocardiographic detection may be related to tumor size [12]. In addition, tumor location appears to be an important factor, because the available data indicate that HSAs located in the RAu are significantly less likely to be detected by echocardiography. The RAu is usually not visualized in normal dogs by ordinary ultrasound imaging techniques, suggesting that relatively small tumors in this area may be difficult to detect [12]. In any event, the sensitivity of echocardiography determined in this study suggests that this technique is much more effective for detection of cardiac masses than has been considered previously $[2,9,12,17,22]$. Technological advances have led to better greyscale resolution, and an improvement of sensitivity for detection of masses may be possible with newer instrumentation [12].

With regard to the treatment of primary cardiac HSA, many dog owners may elect palliation with pericardiocentesis alone, because many affected dogs have evidence of metastasis at the time of diagnosis $[6,30]$. Pericardiocentesis is predictably associated with marked clinical improvement, but clinical signs of cardiac tamponade typically recur within a few days, often resulting in death or prompting euthanasia [30]. If identified early without gross evidence of distant metastasis, a tumor mass can sometimes be removed surgi- 
cally, particularly if it is localized to the RAu [10]. Resection of an RA mass has been reported for treatment of cardiac HSA; however, it has not been shown to improve survival in comparison with either pericardectomy alone or followup without surgical treatment [1, 4, 28, 33]. Dunning et al. [9] reported a median survival time of 16 days in dogs with cardiac HSA following pericardectomy and mass resection. In contrast, a recent study of 23 dogs that underwent median sternotomy or lateral thoracotomy for pericardectomy and mass resection revealed promising results; the mean and median survival times of the 15 dogs treated in this way without adjuvant chemotherapy, were 46 and 42 days, respectively [33]. In the present study, a combination of pericardectomy and surgical resection of the tumor (group C) was associated with significantly longer survival time, compared with no treatment (group A) or medical management alone (group B). Thus, our limited experience suggests that thoracotomy and surgical treatment have the potential to improve the survival time of dogs with cardiac HSA. However, it might be difficult to readily evaluate and compare the effects of surgical treatment with those reported in previous studies, because our group $\mathrm{C}$ included no dogs with stage-3 tumors.

Chemotherapy in an adjuvant setting has been evaluated in various clinical studies of dogs with HSA and has shown promise for prolonging life after surgical resection of the primary HSA lesion [8, 18, 21, 31]. Doxorubicin-based protocols reportedly have moderate efficacy for dogs with HSA, whereas protocols that do not include doxorubicin have had limited or no efficacy [30]. In a study of 23 dogs with primary cardiac HSA, the mean survival time for 8 dogs that received chemotherapy after resection of the mass was 164 days, compared with 46 days for 15 dogs that did not receive such chemotherapy [33]. Therefore, it appears that some postoperative adjuvant chemotherapy protocols have the potential to prolong survival in comparison with surgery alone. In the present study, 5 of the 51 dogs received adjuvant chemotherapy after pericardectomy and tumor resection. Those dogs showed significantly longer survival than the 12 dogs that did not receive chemotherapy after surgery. The mean survival time of 183 days for the above 5 dogs in this study is compatible with that reported by Weisse et al. [33].

The chemotherapy protocol used in all 5 dogs included doxorubicin, cyclophosphamide and vincristine. The most important reason for this choice was its known efficacy in therapeutic trials with human soft tissue sarcoma [25]. Although only a small number of dogs in this series underwent adjuvant chemotherapy with doxorubicin, cyclophosphamide and vincristine, we conclude that such chemotherapy after surgery may be effective for dogs with primary cardiac HSA.

In conclusion, among 51 dogs with cardiac HSA reported in this series, the Maltese and Miniature Dachshund breeds, as well as the GR, were represented more frequently than other breeds. In most cases, the tumor was localized to the RAu and RA, as reported previously. The lower echocardiographic detection rate of masses located in the RAu compared with those in the RA may have been related to tumor size and/or location. The significantly longer survival time for dogs with cardiac HSA receiving adjuvant chemotherapy indicates that postoperative chemotherapy would probably be useful in this setting.

\section{REFERENCES}

1. Aronsohn, M. 1985. Cardiac hemangiosarcoma in the dog: A review of 38 cases. J. Am. Vet. Med. Assoc. 187: 922-926. [Medline]

2. Berg, R. J. and Wingfield, W. 1984. Pericardial effusion in the dog: A review of 42 cases. J. Am. Anim. Hosp. Assoc. 20: 721-730.

3. Boston, S. E., Higginson, G. and Monteith, G. 2011. Concurrent splenic and right atrial mass at presentation in dogs with HSA: a retrospective study. J. Am. Anim. Hosp. Assoc. 47: 336-341. [Medline] [CrossRef]

4. Brown, N. O., Patnaik, A. K. and MacEwen, E. G. 1985. Canine hemangiosarcoma. J. Am. Vet. Med. Assoc. 186: 56-58. [Medline]

5. Burke, A. P. and Virmani, R. 2001. Tumors and tumor-like conditions of the heart. pp. 583-605. In: Cardiovascular Pathology, 3rd ed. (Silver, M. D., Gotlieb, A. I. and Schoen, F. I. eds.), Churchill Livingstone, New York.

6. Crumbaker, D. M., Rooney, M. B. and Case, J. B. 2010. Thoracoscopic subtotal pericardiectomy and right atrial mass resection in a dog. J. Am. Vet. Med. Assoc. 237: 551-554. [Medline] [CrossRef]

7. de Madron, E., Helfand, S. C. and Stebbins, K. E. 1987. Use of chemotherapy for treatment if cardiac hemangiosarcoma in a dog. J. Am. Vet. Med. Assoc. 190: 887-891. [Medline]

8. Dervisis, N. G., Dominguez, P. A., Newman, R. G., Cadile, C. D. and Kitchell, B. E. 2011. Treatment with DAV for advancedstage hemangiosarcoma in dogs. J. Am. Anim. Hosp. Assoc. 47: 170-178. [Medline] [CrossRef]

9. Dunning, D., Monnet, E., Orton, E. C. and Salman, M. D. 1998. Analysis of prognostic indicators for dogs with pericardial effusion: 46 cases (1985-1996). J. Am. Vet. Med. Assoc. 212: 1276-1280. [Medline]

10. Eyster, G. E. 1999. Basic cardiovascular surgery and procedures. pp. 866-881. In: Textbook of Canine and Feline Cardiology, 2nd ed. (Fox, P. R., Sisson, D. and Moise, N. S. eds.), WB Saunders, Philadelphia.

11. Fernández-del Palacio, M. J., Lopez, J. T., Bayon del Rio, A., Seva Alcaraz, J., Pallarés, F. J. and Martinez, C. M. 2006. Left ventricular outflow obstruction secondary to hemangiosarcoma in a dog. J. Vet. Intern. Med. 20: 687-690. [Medline] [CrossRef]

12. Fruchter, A. M., Miller, C. W. and O'Grady, M. R. 1992. Echocardiographic results and clinical considerations in dogs with right atrial/auricular masses. Can. Vet. J. 33: 171-174. [Medline]

13. Girard, C., Helie, P. and Odin, M. 1999. Intrapericardial neoplasia in dogs. J. Vet. Diagn. Invest. 11: 73-78. [Medline] [CrossRef]

14. Hammer, A. S., Couto, C. G., Filppi, J., Getzy, D. and Shank, K. 1991. Efficacy and toxicity of VAC chemotherapy (vincristine, doxorubicin, and cyclophosphamide) in dogs with hemangiosarcoma. J. Vet. Intern. Med. 5: 160-166. [Medline] [CrossRef]

15. Holt, D., Van Winkle, T., Schelling, C. and Prymak, C. 1992. Correlation between thoracic radiographs and postmortem findings in dogs with hemangiosarcoma: 77 cases (1984-1989). $J$. Am. Vet. Med. Assoc. 200: 1535-1539. [Medline]

16. Jarplid, B. 1964. Studies on the site of leukotic and preleukotic changes in the bovine heart. Path. Vet. 1: 366-408. [CrossRef]

17. Kerstetter, K. K., Krahwinkel, D. J. Jr., Millis, D. L. and Hahn, 
K. 1997. Pericardiectomy in dogs: 22 cases (1978-1994). J. Am. Vet. Med. Assoc. 211: 736-740. [Medline]

18. Kim, S. E., Liptak, J. M., Gall, T. T., Monteith, G. J. and Woods, J. P. 2007. Epirubicin in the adjuvant treatment of splenic hemmangiosarcoma in dogs: 59 cases (1997-2004). J. Am. Vet. Med. Assoc. 231: 1550-1557. [Medline] [CrossRef]

19. Kleine, L. J., Zook, B. and Munson, T. O. 1970. Primary cardiac hemangiosarcomas in dogs. J. Am. Vet. Med. Assoc. 157: 326-337. [Medline]

20. Lamerato-Kozicki, A. R., Helm, K. M., Jubala, C. M., Cutter, G. C. and Modiano, J. F. 2006. Canine hemangiosarcoma originates from hematopoietic precursors with potential for endothelial differentiation. Exp. Hematol. 34: 870-878. [Medline] [CrossRef]

21. Lana, S., U'ren, L., Plaza, S., Elmslie, R., Gustafson, D., Morley, P. and Dow, S. 2007. Continuous low-dose oral chemotherapy for adjuvant therapy of splenic hemangiosarcoma in dogs. J. Vet. Intern. Med. 21: 764-769. [Medline] [CrossRef]

22. MacDonald, K. A., Cagney, O. and Magne, M. L. 2009. Echocardiographic and clinicopathologic characterization of pericardial effusion in dogs: 107 cases (1985-2006). J. Am. Vet. Med. Assoc. 235: 1456-1461. [Medline] [CrossRef]

23. Oksanen, A. 1978. Hemangiosarcpma in dogs. J. Comp. Pathol. 88: 585-595. [Medline] [CrossRef]

24. Pearson, G. R. and Head, K. W. 1976. Malignant hemangioendothelioma (angiosarcoma) in the dog. J. Small Anim. Pract. 17: 737-745. [Medline] [CrossRef]

25. Russell, W. O., Cohen, J., Enzinger, F., Hajdu, S. I., Heise, H., Martin, R. G., Meissner, W., Miller, W. T., Schmitz, R. L. and Suit, H. D. 1977. A clinical and pathological staging system for soft tissue sarcomas. Cancer 40: 1562-1570. [Medline] [CrossRef]
26. Snyder, J. M., Lipitz, L., Skorupski, K. A., Shofer, F. S. and Van Winkle, T. J. 2008. Secondary intracranial neoplasia in the dog: 177 cases (1986-2003). J. Vet. Intern. Med. 22: 172-177. [Medline] [CrossRef]

27. Srebernik, N. and Appleby, E. C. 1991. Breed prevalence and sites of haemangioma and haemangiosarcoma in dogs. Vet. Rec. 129: 408-409. [Medline] [CrossRef]

28. Stafford Johnson, M., Martin, M., Binns, S. and Day, M. J. 2004. A retrospective study of clinical findings, treatment and outcome in 143 dogs with pericardial effusion. J. Small Anim. Pract. 45 546-552. [Medline] [CrossRef]

29. Thamm, D. M. 2007. Miscellaneous tumors. pp. 785-795. In: Small Animal Clinical Oncology, 4th ed. (Withrow, S. J. and Vail, D. M. eds.), Saunders, St. Louis.

30. Tobias, A. H. and McNiel, E. A. 2008. Pericardial disorders and cardiac tumors. pp. 200-214. In: Manual of Canine and Feline Cardiology, 4th ed. (Tilley, L. P., Smith, F. W. K. Jr., Oyama, M. A. and Sleeper, M. M. eds.), Saunders Elsevier, Philadelphia.

31. U'Ren, L. W., Biller, B. J., Elmslie, R. E., Thamm, D. H. and Dow, S. W. 2007. Evaluation of a novel tumor vaccine in dogs with hemangiosarcoma. J. Vet. Intern. Med. 21: 113-120. [Medline] [CrossRef]

32. Ware, W. A. and Hopper, D. L. 1999. Cardiac tumors in dogs: 1982-1995. J. Vet. Intern. Med. 13: 95-103. [Medline] [CrossRef]

33. Weisse, C., Soares, N., Beal, M. W., Steffey, M. A., Drobatz, K. J. and Henry, C. J. 2005. Survival times in dogs with right atrial HS treated by means of surgical resection with or without adjuvant chemotherapy: 23 cases (1986-2000). J. Am. Vet. Med. Assoc. 226: 575-579. [Medline] [CrossRef] 